\title{
Role of the microRNA 181 family in glioma development
}

\author{
LIU YANG, YUEYUN MA, YIJUAN XIN, RUOBIN HAN, RUI LI and XIAOKE HAO \\ Clinical Laboratory Medicine Center of PLA, Xijing Hospital, \\ Fourth Military Medical University, Xi'an, Shaanxi 710032, P.R. China
}

Received November 8, 2015; Accepted November 18, 2016

DOI: $10.3892 / \mathrm{mmr} .2017 .7895$

\begin{abstract}
MicroRNAs (miRNAs) are small, noncoding regulatory RNAs that regulate protein expression by reducing mRNA stability and/or translation, via base pairing with full or partial sequence-complementary target mRNAs. Recent evidence indicates that miRNAs have roles as tumor suppressors and oncogenes. The members of the miRNA181 (miR181) family have been reported to be downregulated in early stage human glioma, and to be involved in glioma development. The current study demonstrated that all subtypes of the miRNA 181 family were downregulated at stages of human glioma, including miR181a1, a2, b1, b2, c and d. In the present study, the family members were detected by reverse transcription-quantitative polymerase chain reaction in glioma tissues of different stages. miR181c declined the most in the samples from patients with World Health Organization (WHO) grade I glioma. As glioma development progressed from grade I to IV, the expression of miRN181 family members continued to decline, with miR181b1 exhibiting the fastest decline rate. Furthermore, a lentivirus was used to overexpress miR181c in primary glioma cells; the result indicated that miR181c overexpression was able to significantly inhibit glioma cell proliferation. Thus, miR181 may be a useful biomarker for human glioma at early stages. Detection of the level of miR181 family members may be a potential method for glioma diagnosis, determining the tumor WHO grade and guiding clinical treatment.
\end{abstract}

\section{Introduction}

Glioma originates from glial cells in the brain and the most common intracranial tumor. In the last 30 years, the incidence of primary malignant brain tumors has increased annually with an annual growth rate of $\sim 1.2 \%$, particularly in the elderly population (1). According to the statistics of the Central Brain Tumor Registry of the United States, malignant gliomas

Correspondence to: Dr Xiaoke Hao, Clinical Laboratory Medicine Center of PLA, Xijing Hospital, Fourth Military Medical University, 15 Changle West Road, Xi'an, Shaanxi 710032, P.R. China E-mail: hao_xiaoke@126.com

Key words: microRNA 181 family, glioma, early stage, alteration, cancer stem cells account for $\sim 70$ percent of primary malignant brain tumors. The annual occurrence rate is $\sim 5$ in 10,000 individuals, with $>14,000$ newly diagnosed each year, and incidence is significantly higher in people over the age of 65 (2). The annual mortality rate from glioma has risen in China to 30,000. The characteristics of glioblastoma include invasive growth, infiltration in to normal brain tissue without clear boundaries, fast progression and short survival time. Typically, gliomas are not limited to one lobe of the brain; the tumor infiltrates and damages the surrounding brain tissue (3). Currently, there is no effective treatment to combat glioma. For the conventional treatment of gliomas in China and other countries, surgery, radiotherapy, chemotherapy, stereotactic photon therapy system and stereotactic gamma ray radiation therapy are used (4). According to follow-up and investigation, the recurrence time of World Health Organization (WHO) grade III and/or IV glioma was between 1-6 months, and for grade I-II level recurrence typically occur during 1-2 years after surgery. Following comprehensive treatment of patient with low-grade gliomas (WHO grade I-II grade), the median survival time is between 8-10 years; for patients with aplastic glioma (WHO grade III), the median survival time is 3-4 years; for patients with glioblastoma (WHO grade IV), the median survival is only 14.6-17 months (2).

MicroRNAs (miRNAs) have various forms at different stages of biogenesis. The initial form is pri-miRNA, which is 300-1,000 bases in length. Following primary processing, the pri-miRNA becomes a pre-miRNA, also termed microRNA precursor, of 70-90 bases in length. Pre-miRNA is processed further through digestion by Dicer enzyme, creating a small single-stranded miRNA molecule of 21-23 bases in length. miRNAs are different from, but closely associated with small interfering RNA (siRNA) duplexes (5). The majority of miRNA genes are present in the genome asa single copy, multiple copies or gene clusters $(5,6)$. For use in research, pre-miRNAs are the most commonly used miRNA form. Numerous commercial miRNA libraries contain RNAs in the pre-miRNA form. A previous study demonstrated that miRNA arms serve an important role in the formation of mature miRNA, thus, pri-miRNAs that retain the miRNA arms are increasingly being adopted for use by researchers (3).

Presumably, these non-coding small molecule RNAs are involved in the regulation of gene expression, however its mechanism is different from siRNA-mediated mRNA degradation. The first characterized miRNAs were lin-4 and let-7, which were discovered in nematodes (7). Subsequently, 
hundreds of miRNAs were identified in various species, including in humans, fruit flies and plants by numerous research teams. miRNAs are involved in transcriptional regulation of gene expression in plants and animals and have a variety of important roles in the cell. Scientists previously identified 28,645 miRNA molecules in viruses, plants and animals (8). The majority of identified miRNAs have a hairpin structure. Single stranded RNA hairpin precursorsof $\sim 70$ bases are generated through Dicer enzyme processing, and a 5 ' end phosphate group and a 3 ' hydroxyl group are located at the $3^{\prime}$ or $5^{\prime}$ end of the RNA precursor, respectively (9). Each miRNA may have multiple target genes, and the expression of one gene may be regulated by a combination of several miRNAs resulting in a complex regulatory network (10). Potentially, miRNAs may regulate the expression of a third of human genes. The genes that encode miRNAs are always located in transcription units (11), the majority of which are located in intronic regions (12). The position of miRNA genes in introns is highly conserved among different species. miRNA genes are not only conserved in position, but also have high sequence homology (13-15). The high conservation of miRNAs is closely associated with their functions. miRNAs are also closely associated with the evolution of their target genes, therefore, examining the evolutionary history of miRNAs may help to understand the mechanism of action and functions of miRNAs (9).

miRNAs have many biological functions in glioma, including modulating glioma cell apoptosis and tumor angiogenesis, and therefore, modulating glioma cell proliferation, invasion, radio-resistance and migration (1). miRNAs are also involved in glioma stem cell development and maintenance, and contribute to glioma resistance to therapies. Briefly, miRNAs act as 'gap fillers', 'amplifiers', 'fine-tuners' and 'crosstalk mediators' in different glioma-associated cellular signaling networks, demonstrating the biological importance of miRNAs in glioma (1).

miRNA (miR)181a, miR181b and miR181c were originally identified as downregulated miRNAs in glioblastoma cells and tumors by miRNA microarrays (16). miR181a, and to a greater extent miR181b, were subsequently described as tumor suppressors that inhibit growth and induce apoptosis of glioma cells (17). miR181a overexpression sensitizes glioma cells to radiation treatment concurrent with the downregulation of Bcl-2 expression (18). Also, miR181b and miR181c were reported to be significantly downregulated in patients that responded to radiation therapy and temozolomide compared with patients with progressive disease. Therefore, it was proposed that expression levels of miR $181 \mathrm{~b}$ and miR181c may serve as a predictive marker for response to radiation therapy and temozolomide in patients with glioblastoma (19).

\section{Materials and methods}

Samples. Samples from 20 patients were used in the current study. Samples were collected between March 2015 and February 2016 from the Center for Clinical Laboratory Medicine of PLA, Xijing Hospital (Xi'An, China). There were five samples per group as follows: Grade I, primary Grade I pilocyticastrocytomas, infiltrated growth in the cerebral white matter (WHO I); Grade II astrocytoma, neoplastic cells with
Table I. Clinical information of the 20 patients.

\begin{tabular}{lcll}
\hline Patient (group) & Age & Sex & Lesion location \\
\hline 1 (WHO I) & 63 & Male & Right frontal lobe \\
2 (WHO I) & 55 & Female & Right frontal lobe \\
3 (WHO I) & 61 & Female & Left frontal lobe \\
4 (WHO I) & 54 & Male & Left frontal lobe \\
5 (WHO I) & 49 & Female & Right frontal lobe \\
6 (WHO II) & 58 & Male & Left frontal lobe \\
7 (WHO II) & 57 & Male & Right frontal lobe \\
8 (WHO II) & 48 & Female & Right frontal lobe \\
9 (WHO II) & 63 & Female & Left frontal lobe \\
10 (WHO II) & 45 & Male & Left frontal lobe \\
11 (WHO III) & 47 & Male & Right frontal lobe \\
12 (WHO III) & 56 & Female & Right frontal lobe \\
13 (WHO III) & 48 & Female & Left frontal lobe \\
14 (WHO III) & 62 & Female & Left frontal lobe \\
15 (WHO III) & 60 & Male & Right frontal lobe \\
16 (WHO IV) & 51 & Male & Left frontal lobe \\
17 (WHO IV) & 59 & Female & Right frontal lobe \\
18 (WHO IV) & 46 & Male & Right frontal lobe \\
19 (WHO IV) & 57 & Female & Left frontal lobe \\
20 (WHO IV) & 64 & Male & Left frontal lobe \\
\hline
\end{tabular}

WHO, World Health Organisation.

almost unity nucleus and perfect differentiation, widespread growth, thus, evident pathological changes in the microcapsule (WHO II); Grade III anaplastic astrocytomas, neoplastic cells with pleomorphism, cacoethic differentiation, karyokinesis, capillary vessel generation (WHO III); Grade IV glioblastoma multiforme, neoplastic cells have pleomorphism, high density, undifferentiation, capillary vessel generation, coagulation necrosis (WHO IV); and matched normal brain tissues derived from the temporal lobes and saddle area $2 \mathrm{~cm}$ away from tumor tissue (Table I). The average of the ages of the WHO I to IV group were $56.4 \pm 2.5,54.2 \pm 3.3,54.6 \pm 3.1$ and $55.4 \pm 3.1$ respectively. There were 5 patients in each group (M: F ratio: WHO I, 2:3; WHO II, 3:2; WHO III, 2:3; WHO IV, 3:2). Each sample was divided into two parts, one was used for RNA extraction (TRIzol method) and the other was used for primary cell culture (tissues were immersed in DMEM, and maintained on ice for a maximum of $4 \mathrm{~h}$ ). Informed consent was acquired from every patient prior to participation, and the study was approved by the Ethics committee of First Affiliated Hospital of Fourth Military Medical University (Certificate no. KY20153226-1).

Primary glioma cell isolation and culture. Primary glioma cells were isolated from patients glioma tissue without selectively removing cells (containing all tissue cells). Connective tissue and blood vessels were removed from the tumor. The remaining tissue were dissected into small pieces and digested for $10 \mathrm{~min}$ in $0.25 \%$ trypsin (Invitrogen; Thermo Fisher Scientific, Inc., Waltham, MA, USA). The dissociated cells were cultured in high glucose Dulbecco's modified Eagle's 


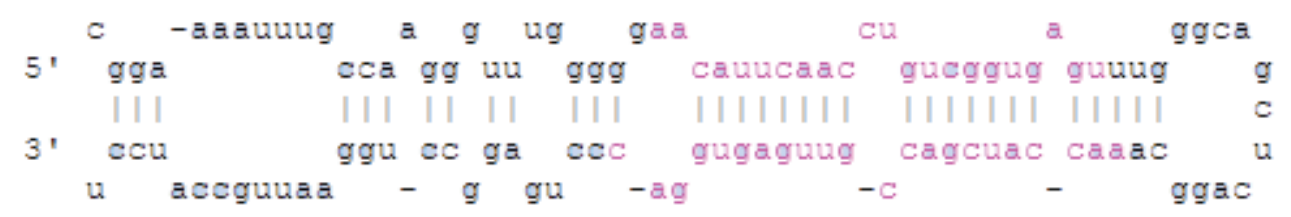

Figure 1. The sequence of miR181c showing the stem-loop structure.

medium (DMEM; Invitrogen; Thermo Fisher Scientific, Inc.), supplemented with $10 \%$ fetal bovine serum (FBS; Biochrom Ltd., Cambourne, UK), $0.1 \mathrm{mM}$ non-essential amino acids and $2 \mathrm{mM}$ Glutamax at $37^{\circ} \mathrm{C}$ and $5 \% \mathrm{CO}_{2}$. Cells became confluent within 2-3 days and were passaged in a 1:4 split. Primary glioma cells were used between passages 2 and 4 . The method of miR181c lentivirus (pCDH-miR181c-GFP) packaging followed the protocol of a previous report (20). Subsequently, the lentivirus was used to infect glioma cells at a multiplicity of infection (MOI; the number of viral particles per cell) of 10. Control cells were transfected with pCDH-GFP. Following infection for 8-12 h, cells were cultured in DMEM with $10 \%$ FBS. The miR181c sequence is presented in Fig. 1.

Reverse transcription-quantitative polymerase chain reaction (RT-qPCR) analysis. TRIzol extraction of total RNA from glioma tissue was performed according to the manufacturer's instructions (Sigma-Aldrich; Merck KGaA, Darmstadt, Germany). Total RNA (500 ng) was reversed transcribed using a miRcute miRNA First Strand cDNA kit (KR201; Tiangen Biotech Co., Ltd., Beijing, China) and then quantified using SYBR Green (Tiangen Biotech, Co., Ltd., FP401) on an ABI 7500 machine (Thermo Fisher Scientific, Inc.). The thermocycling conditions were as follows: $94^{\circ} \mathrm{C} 2 \mathrm{~min}$, followed by 40 cycles of $94^{\circ} \mathrm{C}$ for $40 \mathrm{sec}, 60^{\circ} \mathrm{C}$ for $40 \mathrm{sec}$ and $72^{\circ} \mathrm{C}$ for $1 \mathrm{~min}$. Final extension was performed at $72^{\circ} \mathrm{C}$ for $5 \mathrm{~min}$. PCR miRNA levels were quantified using the $2^{-\Delta \Delta C q}$ method with 3 repeats (21), and $\beta$-actin was used as the reference gene. The following primers (5'-3') were used for qPCR: miR181a1, AGGTTGCTAGGACATCAACGG (F), AGAGTTAGTTTG GTAGCTGGC (R); miR181a2, ATCAGGCCAGCCTTCAAT CT (F), ACTGGCAACTACAGGAACCC (R); miR181b1, TCAACTGGAAGGGTCACACATT (F), ATGTTGTTG GGCGAACCCTC (R); miR181b2, CTGACTCAACTTGTT GGCTGC (F), GTGACTAGTTACTTACACGCCG (R); miR181c, TTTGAGTGGAACTAGGCAGGAC (F), GTAGCT GCAACTCACGGGTC (R); miR181d, GGTGAATGTCCC CTCCCCTA (F), TACTTACAACACCGACCGCC (R); and $\beta$-actin, GGCTGTATTCCCCTCCATCG (F), CCAGTTGGT AACAATGCCATGT (R).

Immunofluorescence. Following overnight culture, Glioma cells were plated at 5,000 cells $/ \mathrm{cm}^{2}$, infected with lentivirues as aforementioned, and then allowed to proliferate for further 5 days. The cells were fixed with $4 \%$ paraformaldehyde (PFA) in $0.1 \mathrm{~mol} / 1 \mathrm{PBS}$ for $20 \mathrm{~min}$ at room temperature and blocked in 5\% BSA with $0.1 \%$ Triton X-100 in PBS for $30 \mathrm{~min}$ at room temperature. Primary antibodies were diluted in antibody dilution solution (PBS with $1 \%$ BSA and $0.1 \%$ Triton X-100) in the following ratios, and secondary antibodies were diluted 1:1,000 in antibody dilute solution. Glioma cells were incubated in primary antibodies overnight at $4{ }^{\circ} \mathrm{C}$ and with secondary antibodies were incubated for $60 \mathrm{~min}$ at room temperature. The following antibodies were used: Rabbit anti-Ki67 (1:500; AB 9260, EMD Millipore, Billerica, MA, USA) and Alexa Cy3-conjugated secondary antibody $(1: 1,000 ; 111-165-003$, Jackson ImmunoResearch Laboratories, Inc.-West Grove, PA, USA). Cells were also stained with DAPI 5 min at room temperature (1:1,000; D9542, Sigma-Aldrich). Proliferation was indicated by $\mathrm{Ki} 67^{+}$-staining and infected cells were detected by observing GFP expression, using an Olympus IX71 microscope (Olympus Corporation, Tokyo, Japan). The number of proliferating infected cells was calculated as the percentage of $\mathrm{Ki} 67^{+}+\mathrm{GFP}^{+}$cells/total $\mathrm{GFP}^{+}$cells. The value was calculated as the average of three parallel wells (24 well) and 10 microscope fields per well.

Ball formation. Glioma cells were suspended at 100 cells $/ \mathrm{ml}$, plated at $1 \mathrm{ml} /$ well into 24 -well plates, and cultured for 5 days. Cells forming ball-like structures were considered to be cancer stem cells. Cells which grew into a colony were selected, digested and separated into single cells and then infected with the miR181c and control lentivirus, and expanded in vitro at 100 cells $/ \mathrm{ml}$ for a further three days. The balling proportion was calculated as the number of balls after 3 days/original cell number. The diameter was calculated using the ruler tool on an Olympus IX71 microscope. The ball diameter and balling proportion was calculated from the average of 3 parallel wells (24 well).

Statistical analysis. Statistical analysis was evaluated using SPSS 16.0 software (SPSS, Inc., Chicago, IL, USA) and assessed with normality and variance. Data were compared by $t$ test, one-way analysis of variance (ANOVA) for multiple comparisons, or two-way ANOVA for repeated-measures, followed by the Games-Howell post hoc test. Data were presented as the mean \pm standard deviation in each experiment. $\mathrm{P}<0.05$ was considered to indicate a statistically significant difference.

\section{Results}

miRNA181 family member expression levels are decreased in glioma. Given the significance of miR181 in glioma, it is important to investigate the role of miR 181 family members in the development and treatment of glioma. The current study examined the expression of miR181a1 in the normal brain tissue from patient with WHO Grade I-IV gliomas. Normal tissue was collected $2 \mathrm{~cm}$ away from the tumor. The results demonstrated that, among all the WHO grades, there was no significant difference in the relative expression of miR181a1 in the adjacent normal tissue (Fig. 2A). This indicated that 

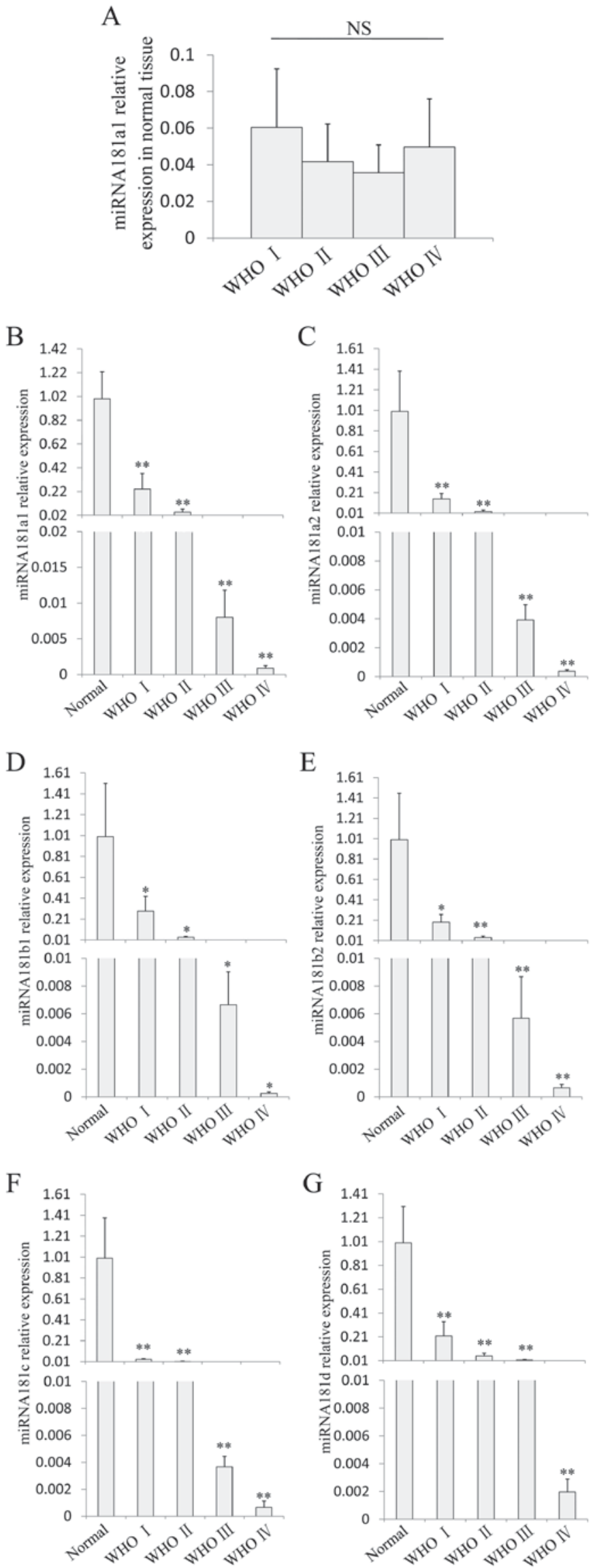

Figure 2. miRNA181 family member expression levels are decreased in glioma. (A) There is no difference between the miRNA181a1 relative expression in the normal tissue adjacent to I-IV grade gliomas. Normal tissue was used as the control and the relative expression of each member of the miRNA181 family, (B) miRNA181a1, (C) miRNA181a2, (D) miRNA181b1, (E) miRNA181b2, (F) miRNA181c and (G) miRNA181d, was reduced significantly in glioma compared with normal tissue, and further decreased with the gradual progress of glioma. ${ }^{*} \mathrm{P}<0.05,{ }^{* *} \mathrm{P}<0.01$, vs. normal; NS, P $>0.1$. miRNA, microRNA; WHO, World Health Organization grade. 


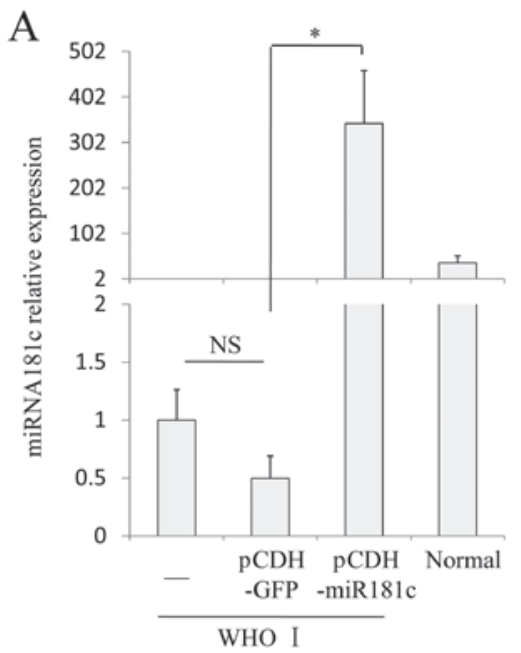

B
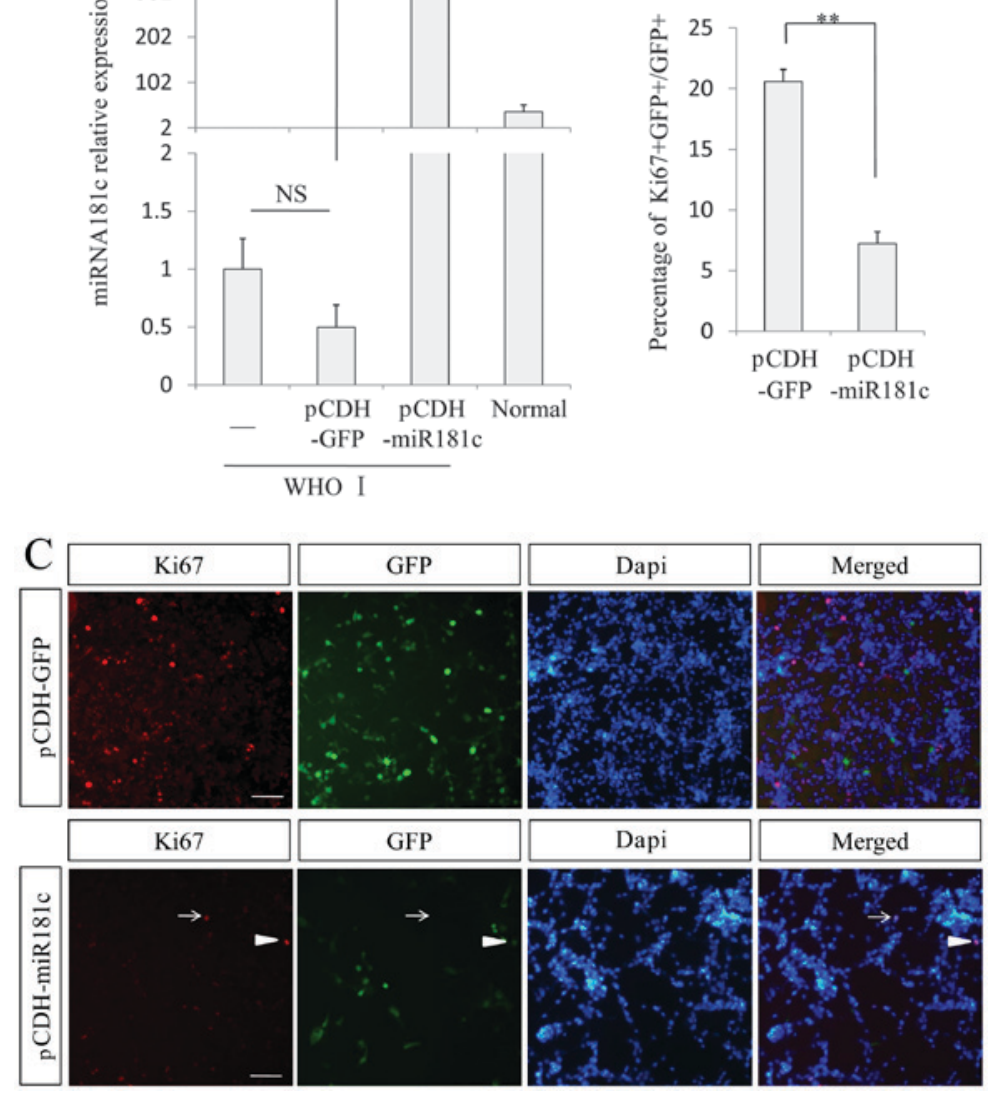

Figure 3. miR181c inhibits glioma WHO Grade I tumor cell proliferation. (A) miR181c was overexpressed in the WHO I glioma cells using a lentivirus (pCDH-miR181c). The relative miR181c expression level reached to normal tissues level following infection with pCDH-miR181c. (B) In virus-infected cells, relative to the control lentivirus group (pCDH-GFP), in cells overexpressing miRNA181c the number of Ki67-postive cells was significantly reduced. (C) There was no difference inKi67 staining between the $\mathrm{GFP}^{+}$cells and $\mathrm{GFP}^{-}$cells. The proportion of Ki67 + $\mathrm{GFP}^{+}$cells was decreased in the pCDH-miR181c group compared with pCDH-GFP. The proportion of proliferating GFP-cells was not different between $\mathrm{pCDH}-\mathrm{miR} 181 \mathrm{c}$ and the control. The arrow indicates Ki67 + GFP cells, triangle indicates Ki67 $7^{+}+\mathrm{GFP}^{+}$cells. ${ }^{*} \mathrm{P}<0.05,{ }^{* *} \mathrm{P}<0.01$, comparisons indicate by brackets; NS, $\mathrm{P}>0.1$. Scale bars: $100 \mu \mathrm{m}$. miR, microRNA; NS, no significance; WHO, World Health Organization grade.

the expression of the miRNA181 family is unaffected in the normal tissue outside of tumor. Therefore, 1-2 patients in each Grade (I-IV) were selected; a total of five people, and the expression of each miRNA181 subtype was compared with the expression in normal brain tissue. The results demonstrated that the expression of all miR181 subtypes (miR181a1, Fig. 2B; miR181a2, Fig. 2C; miR181b1, Fig. 2D; miR181b2, Fig. 2E; miR181c, Fig. 2F; and miR181d, Fig. 2G) was significantly reduced in glioma compared with the normal tissue $(\mathrm{P}<0.05)$, and the expression level decline was greater with increasing WHO grade.

At the initial period of glioma development (WHO Grade I), the six members of miRNA181 family were already significantly reduced, with all decreased to $<30 \%$ of the levels in the normal tissue. In particular, the miR181c level (Fig. 2F) was markedly decreased in all five of the patients with WHO I grade glioma, with the greatest decrease to $4.2 \%$ of the normal tissue, and an average reduction to $3.3 \pm 0.6 \%$ of normal tissue levels. Expression levels of all members of the miRNA181 family were decreased in the early glioma stage; the expression of miRNA181c declined the most, which has important implications for the early diagnosis and prognosis of glioma. However, during the process of glioma development, the expression of miR181 family members decreased further. As the WHO grade level increased, the expression levels were decreased to $10-30 \%$ of the preceding level. miR181b1 had the fastest decline rate of the family, with the WHO Grade IV expression level even $<0.1 \%$ of WHO Grade I level, and only $0.024 \%$ of the level in normal tissues (Fig. 2D). This result indicates that miRNA181 is critical in glioma and is associated with the level of malignancy; gradually decreasing expression of miRNA181 was accompanied by increasing stage and may be associated with increased degeneration of glial cells.

miR181c inhibits glioma WHO Grade I tumor cell proliferation. Subsequently, it was determined whether miR181 overexpression suppresses glioma cell growth and whether it may be useful for treatment of glioma. Since miR181c exhibited a sharp declined at the glioma initiation stage, miR181c may be an important factor that inhibits glial cell malignant (Fig. 2F). Therefore, a plasmid was constructed to overexpress miR181c, packaged as a lentivirus and infected into primary 

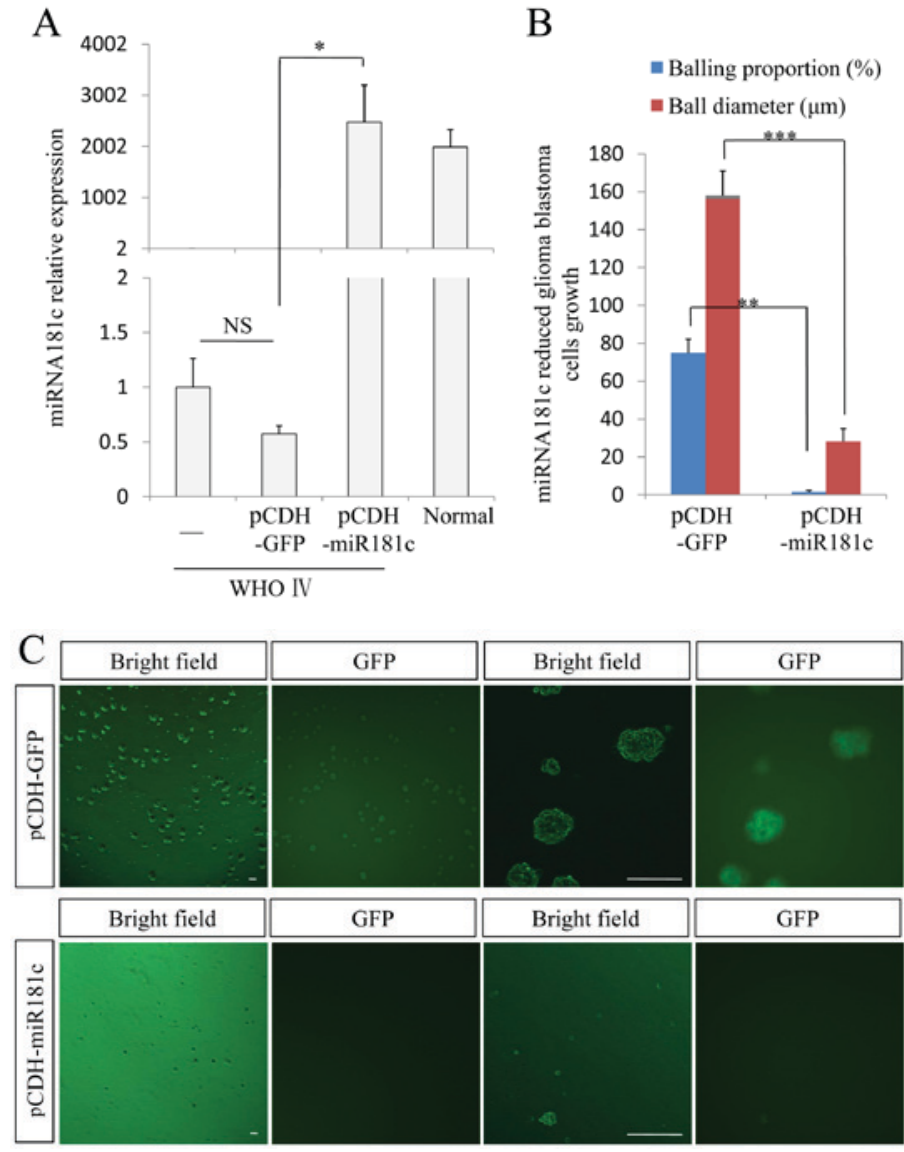

Figure 4. miRNA181c inhibits glioma WHO Grade IV cancer stem cell proliferation. (A) miRNA181c was over expressed in the WHO Grade IV blastoma cells using a lentivirus (pCDH-miRNA181c). The relative expression level of miRNA181c reached normal tissues levels following infection with the lentivirus. (B) In glioma cells cultured in suspension, cells forming ball-like structures were considered to be cancer stem cells. The ability to form balls was significantly decreased in cells overexpressing miRNA181c compared with pCDH-GFP, in the proportion of cells and the ball diameter. (C) In the control group (pCDH-miRNA181c) $75 \%$ of the cells formed balls, with the ball diameter $\sim 160 \mu \mathrm{m}$. Overexpressing miRNA181c reduced the balling proportion to $1-2 \%$, and the ball diameter down to $30 \mu \mathrm{m}$. ${ }^{*} \mathrm{P}<0.05,{ }^{* *} \mathrm{P}<0.01,{ }^{* * *} \mathrm{P}<0.001$, comparison indicated by brackets; NS, $\mathrm{P}>0.1$. Scale bars, $100 \mu \mathrm{m}$. miR, microRNA; NS, no significance; WHO, World Health Organization grade.

glioma cells, to determine the role of miR181c in the different stages of glioma. Initial experiments were performed using WHO Grade I patients cultured in vitro. RT-qPCR was performed to detect expression level of miR181c in normal cells, uninfected glioma cells, control virus glioma cells (pCDH-GFP) and glioma cells overexpressing miR181c. In cells overexpressing miR181c (pCDH-miR181c), the miR181c levels were significantly increased compared with control virus cells ( $\mathrm{P}=0.028)$, and also markedly increased compared with the levels in normal cells (Fig. 3A). However, there was no significant difference between the miR181c levels in cells infected with the control virus compared with the uninfected group. Subsequently, the proliferation ability of these cells was analyzed (22). Cells infected with the control virus proliferated rapidly during the logarithmic growth phase, $>20 \%$ of $\mathrm{GFP}^{+}$cells were proliferative (indicated by $\mathrm{Ki}^{+} 7^{+}$ staining), and the GFP- cells also had similar proliferation rates. Overexpression of miR181c in WHO Grade I glioma cells significantly decreased cell proliferation compared with cells infected with the control virus $(\mathrm{P}=0.0097$; Fig. 3B and C). Following culture for the equivalent time (48 h), the cells number (Dapi quantity) was lower in the miR181c overexpressing cells than the control virus-infected group cells, and the proportion of $\mathrm{GFP}^{+}-\mathrm{Ki} 67^{+}$cells was $7.21 \pm 0.56 \%$, significantly lower than the proportion in the infection control virus group (Fig. 3B and C).

miRNA181c inhibits glioma WHO Grade IV cancer stem cell proliferation. Subsequently, the current study investigated whether miR181c also has an inhibitory effect on gliomablastoma cells. As gliomablastoma cells have stem cell properties, colonies can form as balls in suspension culture; cells that form balls within a low-density suspension culture are regarded as gliomablastoma cells (23). Therefore, the cells from patients with WHO Grade IV glioma were cultured in suspension. Cells that grew into a colony were selected, digested and separated into single cells, and then infected with the miR181c and control lentivirus. The virus titer was adjusted to MOI=10. Initially, whether the infection of the gliomablastoma cells with the miR181c lentivirus restored the expression of miR181c was determined by RT-qPCR. The results demonstrated that miR181c expression was lower in gliomablastoma cells than in Grade IV glioma cells, and after infection of blastoma cells with pCDH-miR181c, the levels of miR181c were significantly increased compared with pCDH-GFP cells $(\mathrm{P}=0.027)$, and comparable to normal cell levels (Fig. 4A). Subsequently, the secondary ball formation ability the infected cells was evaluated. Cells were cultured at a starting density of 100 cells $/ \mathrm{ml}$ 
in 24-well plates for $72 \mathrm{~h}$. The number of balls per well as the proportional to form ball, and the ball diameter was measured. The results demonstrated that the balling proportion of cells infected with the control virus was $74.9 \pm 7.35 \%$ and the mean diameter of the ball was $158 \pm 13 \mu \mathrm{m}$, which indicated that $\sim 75 \%$ of cells had cancer stem cell properties and a high proliferation rate (Fig. 4B and C). However, the cell proliferation was decreased by infection with the miR181c virus compared with the control virus, and the balling proportion and ball diameter were $1.53 \pm 0.8 \%$ and $28.2 \pm 6.65 \mu \mathrm{m}$, respectively ( $\mathrm{P}=0.0030$ and $\mathrm{P}=0.00063$ vs. $\mathrm{pCDH}-\mathrm{GFP}$, respectively). The results demonstrated that restoration of miRNA181c expression appeared to reverse the tumor cell (and also tumor stem cell) phenotype, by reducing excessive proliferation, and restoring regular properties observed in normal glial cells (Fig. 4B and C).

\section{Discussion}

miRNA181 is an ancient miRNA gene family that originated in urochordata. miRNAs are endogenously expressed small non-coding RNAs (22 nucleotides) that regulate the expression of protein-coding genes post-transcriptionally. They predominantly function by base-pairing to the 3-untranslated regions of mRNAs to induce translational inactivation or RNA degradation. As a class of important gene regulators throughout the evolutionary process, miRNA-meditated gene regulation is widespread in animals, plants and viruses. Previous studies have demonstrated the involvement of the hsa-miR-181 family in gliomaoncogenesis, and miR181 expression was typically downregulated in glioma tissue (17-19). Hsa-miR-181a and hsa-miR-181b functioned as tumor suppressors that inhibited growth, induced apoptosis and reduced invasion in glioma cells. Furthermore, the tumor-suppressive effect of hsa-miR-181b in glioma cells was more apparent than the effect of hsa-miR-181a, which suggested that aberrantly downregulated hsa-miR-181a and hsa-miR-181b may be critical factors that contribute to the malignancy of human gliomas. Previous studies reported that miR-21 and 221 were overexpressed in glioma samples, whereas miRNA 181 family members were downregulated compared with normal brain tissue $(1,24)$. These findings suggest that aberrant downregulation of miR-181a/miR-181b and miR-181c could serve as a predictive marker that may contribute to malignant appearance in human gliomas $(17,19)$. Slaby et al (19) and Zhang et al (25) demonstrated that the expression signature of miR-181 family members may be useful in predictive glioblastoma biomarkers.

However, to the best of our knowledge, no detailed analysis of specific trends within different glioma stages has been performed. Thus, the current study determined the expression trends of the six subtypes of the miR 181 family in detail and evaluated the changes in their expression in the four WHO glioma stages. Additionally, the effects of miR181 on glioma cell proliferation and malignancy were investigated. The current study demonstrated that restoring the expression of miR $181 \mathrm{c}$ reduced the proliferation of glioma cells in the early or late stage (Grade I and Grade IV) and in gliomablastoma cells, restoring normal glia cells characteristics. Therefore, miR181 has important implications for the diagnosis of glioma, and also may be useful as a therapeutic method.
The results of the current study demonstrated that the expression level of all six subtypes of miR181 were decreased in glioma compared with normal tissue, particularly miR181c, which exhibited the greatest decrease. With the progression of gliomas from Grade I to IV, the expression levels of the miRNAs also decreased, and miR181b1 decreased the fastest. Thus, the expression level changes of the different miR181 subtypes are important for the early diagnosis of glioma and for predicting prognosis.

As the miR181c expression level was greatly decreased at the early stage of glioma, and continued to decline by $>1,000$-fold with the development of glioma (to Grade IV), miR181c maybe a crucial factor involved in the glioma cell transformation and glioma progression. Thus, the effects of miR181c on glioma cell proliferation and malignancy were also examined. The present study demonstrated that, in the early and late stage, miR181c expression recovery reduced the proliferation of glioma cells and gliomablastoma cells, restoring normal glia cells characteristics to these cells. Therefore, miR181 has important implications for the diagnosis of glioma, and may also be useful as a therapeutic for glioma treatment.

\section{References}

1. Li M, Li J, Liu L, Li W, Yang Y and Yuan J: MicroRNA in human glioma. Cancers (Basel) 5: 1306-1331, 2013.

2. Kohler BA, Ward E, McCarthy BJ, Schymura MJ, Ries LA, Eheman C, Jemal A, Anderson RN, Ajani UA and Edwards BK: Annual report to the nation on the status of cancer, 1975-2007, featuring tumors of the brain and other nervous system. J Natl Cancer Inst 103: 714-736, 2011.

3. Floyd D and Purow B: Micro-masters of glioblastoma biology and therapy: Increasingly recognized roles for microRNAs. Neuro Oncol 16: 622-627, 2014.

4. Ostrom QT, Bauchet L, Davis FG, Deltour I, Fisher JL, Langer CE, Pekmezci M, Schwartzbaum JA, Turner MC, Walsh KM, et al: The epidemiology of glioma in adults: A 'state of the science' review. Neuro Oncol 16: 896-913, 2014.

5. Aguirregomoscorta Urquijo JI, Menéndez Gastan I, Aparicio Abendaño S, Quintana López J, Capelastegui Saiz A and Urrutia Landa I: Nap polysomnography: Sufficient grounds for initiating CPAP treatment?. Arch Bronconeumol 37: 302-306, 2001.

6. Lau NC, Lim LP, Weinstein EG and Bartel DP: An abundant class of tiny RNAs with probable regulatory roles in Caenorhabditis elegans. Science 294: 858-862, 2001.

7. Bagga S, Bracht J, Hunter S, Massirer K, Holtz J, Eachus R and Pasquinelli AE: Regulation by let-7 and lin-4 miRNAs results in target mRNA degradation. Cell 122: 553-563, 2005.

8. Ha M, Pang M, Agarwal V and Chen ZJ: Interspecies regulation of microRNAs and their targets. Biochim Biophys Acta 1779: 735-742, 2008.

9. Gurtan AM, Lu V, Bhutkar A and Sharp PA: In vivo structure-function analysis of human Dicer reveals directional processing of precursor miRNAs. RNA 18: 1116-1122, 2012.

10. Bao W, Greenwold MJ and Sawyer RH: Expressed miRNAs target feather related mRNAs involved in cell signaling, cell adhesion and structure during chicken epidermal development. Gene 591: 393-402, 2016.

11. Rodriguez A, Griffiths-Jones S, Ashurst JL and Bradley A: Identification of mammalian microRNA host genes and transcription units. Genome Res 14: 1902-1910, 2004.

12. Kim Y, Nam YJ and Lee C: Haplotype analysis of single nucleotide polymorphisms in VEGF gene for vascular dementia. Am J Med Genet B Neuropsychiatr Genet 141B: 332-335, 2006.

13. Pasquinelli AE, Reinhart BJ, Slack F, Martindale MQ, Kuroda MI, Maller B, Hayward DC, Ball EE, Degnan B, Müller P, et al: Conservation of the sequence and temporal expression of let-7 heterochronic regulatory RNA. Nature 408: 86-89, 2000.

14. Ruvkun G: Molecular biology. Glimpses of a tiny RNA world. Science 294: 797-799, 2001.

15. Lee RC and Ambros V: An extensive class of small RNAs in Caenorhabditis elegans. Science 294: 862-864, 2001. 
16. Ciafrè SA, Galardi S, Mangiola A, Ferracin M, Liu CG, Sabatino G, Negrini M, Maira G, Croce CM and Farace MG: Extensive modulation of a set of microRNAs in primary glioblastoma. Biochem Biophys Res Commun 334: 1351-1358, 2005.

17. Shi L, Cheng Z, Zhang J, Li R, Zhao P, Fu Z and You Y hsa-mir-181a and hsa-mir-181b function as tumor suppressors in human glioma cells. Brain Res 1236: 185-193, 2008.

18. Chen G, Zhu W, Shi D, Lv L, Zhang C, Liu P and Hu W: MicroRNA-181a sensitizes human malignant glioma U87MG cells to radiation by targeting Bcl-2. Oncol Rep 23: 997-1003, 2010.

19. Slaby O, Lakomy R, Fadrus P, Hrstka R, Kren L, Lzicarova E, Smrcka M, Svoboda M, Dolezalova H, Novakova J, et al: MicroRNA-181 family predicts response to concomitant chemoradiotherapy with temozolomide in glioblastoma patients. Neoplasma 57: 264-269, 2010.

20. Shen LJ, He JL, Yang DH, Ding YB, Chen XM, Geng YQ, Liu SJ, Liu XQ and Wang YX: Mmu-microRNA-200a overexpression leads to implantation defect by targeting phosphatase and tensin homolog in mouse uterus. Reprod Sci 20: 1518-1528, 2013.
21. Livak KJ and Schmittgen TD: Analysis of relative gene expression data using real-time quantitative PCR and the 2(-Delta Delta C(T)) method. Methods 25: 402-408, 2001.

22. Paulus W: GFAP, Ki67 and IDH1: Perhaps the golden triad of glioma immunohistochemistry. Acta Neuropathol 118: 603-604, 2009.

23. Wirth T: A short perspective on gene therapy: Clinical experience on gene therapy of gliomablastoma multiforme. World J Exp Med 1: 10-16, 2011.

24. Conti A, Aguennouz M, La Torre D, Tomasello C, Cardali S, Angileri FF, Maio F, Cama A, Germanò A, Vita G and Tomasello F: miR-21 and 221 upregulation and miR-181b downregulation in human Grade II-IV astrocytic tumors. J Neurooncol 93: 325-332, 2009.

25. Zhang W, Zhang J, Hoadley K, Kushwaha D, Ramakrishnan V, Li S, Kang C, You Y, Jiang C, Song SW, et al: miR-181d: A predictive glioblastoma biomarker that downregulates MGMT expression. Neuro Oncol 14: 712-719, 2012. 